\title{
Amyotrophic dermatomyositis
}

\author{
Sundeep Shenoy MD, Suman Thapamagar MD
}

Cite as: CMAJ 2017 January 30;189:E164. doi: 10.1503/cmaj.151448

A 50-year-old woman presented with a three-month history of nonpruritic skin rash, weakness and myalgia. The skin rash, which preceded the weakness, started on her face and spread to her back, but spared the mucous membranes. Strength in her bilateral upper and lower extremities was decreased, and the proximal group muscles were tender and very painful on movement. Possible diagnoses included discoid lupus erythematosus, systemic lupus erythematosus and inflammatory myopathies. Serum rheumatoid factor, antinuclear antibodies, creatinine phosphokinase, cortisol and aldolase levels were normal on two occasions; creatinine level was elevated at 144.89 (normal range 50-110) $\mu \mathrm{mol} / \mathrm{L}$.

Pathognomonic skin findings of heliotrope rash, shawl sign, Gottron papules (Figure 1) and periungual erythema suggested amyotrophic dermatomyositis, a variant of dermatomyositis with typical cutaneous features but lacking inflammatory myopathy. ${ }^{1}$ Skin biopsy showed mild, nonspecific interface dermatitis with increased deposition of dermal mucin (Appendix 1, available at www.cmaj.ca/lookup/suppl/doi:10.15 03/cmaj.151448/-/DC1). Despite the patient's muscle pain, creatinine phosphokinase and aldolase levels were normal, and she did not undergo muscle biopsy or electromyography.

There is a fivefold higher incidence of malignancies with dermatomyositis, ${ }^{2}$ and it is likely that the tumour and muscles express common autoantigens. The immune response aimed at tumour cells may also target autoantigens in the muscles, causing muscle inflammation. This makes dermatomyositis a paraneoplastic phenomenon. The cervix, lung, pancreas, ovaries and breast are common sites for such malignancies. ${ }^{3}$ All patients with newly diagnosed dermatomyositis should be evaluated for underlying malignancy. 2,3

In this patient, renal ultrasonography (performed because of the elevated creatinine level) showed bilateral hydronephrosis. Subsequent magnetic resonance imaging showed a cervical mass invading the bladder and ureters. Biopsy showed that it was infiltrative squamous cervical carcinoma. The patient had not undergone any prior cervical cytologic screening.

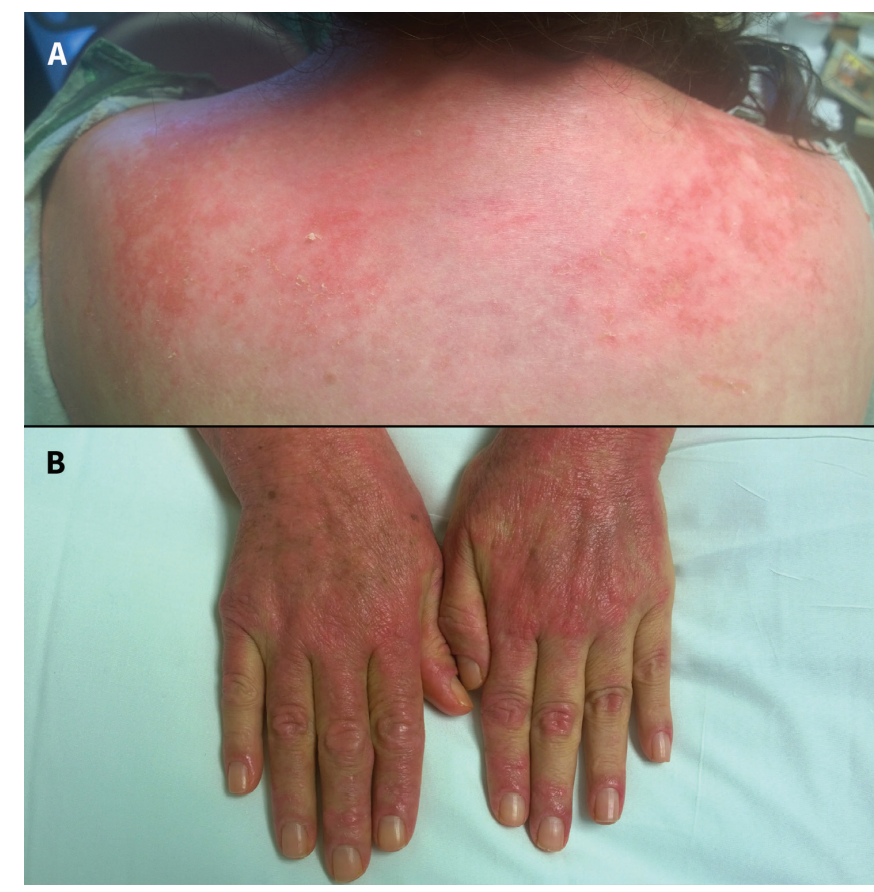

Figure 1: (A) Erythematous eruption on upper back suggests shawl sign. (B) Scaly, violaceous papules over dorsum of the hand suggest Gottron papules.

The patient was treated with carboplatin and paclitaxel for the cervical cancer and oral prednisone for the dermatomyositis. Despite initial improvement in her symptoms, metastases developed in the brain and liver, and there was progresssively worsening renal failure. The patient died about one year after the initial diagnosis.

\section{References}

1. Bailey EE, Fiorentino DF. Amyopathic dermatomyositis: definitions, diagnosis, and management. Curr Rheumatol Rep 2014;16:465.

2. Olazagasti JM, Baez PJ, Wetter DA, et al. Cancer risk in dermatomyositis: a meta-analysis of cohort studies. Am J Clin Dermatol 2015;16:89-98.

3. Femia AN, Vleugels RA, Callen JP. Cutaneous dermatomyositis: an updated review of treatment options and internal associations. Am J Clin Dermatol 2013;14:291-313.

\section{Competing interests: None declared.}

This article has been peer reviewed.

The authors have obtained patient consent.
Affiliation: Inpatient Medicine, University of Arizona, Tucson, Ariz.
Correspondence to: Sundeep Shenoy, sundeepshenoy@email.arizona.edu 